U N IVERSITY OF COPENHAGEN

\title{
Orosensory perception
}

\author{
Møller, Per
}

Published in:

Pediatric formulations

DOI:

10.1007/978-1-4899-8011-3_8

Publication date:

2014

Document version

Publisher's PDF, also known as Version of record

Citation for published version (APA):

Møller, P. (2014). Orosensory perception. In D. Bar-Shalom, \& K. Rose (Eds.), Pediatric formulations: a roadmap (pp. 105-121). Springer. AAPS Advances in the Pharmaceutical Sciences Series Vol. 11 https://doi.org/10.1007/978-1-4899-8011-3_8 


\title{
Chapter 8 \\ Orosensory Perception
}

\author{
Per Møller
}

\begin{abstract}
This chapter provides an introduction to the sensory systems which determine human perception of foods. Since the same sensory systems are stimulated when a patient receives medication via the oral route, properties and effects described in the context of food perception are relevant to the understanding of the perception of pharmacological substances, and these should be taken into account when designing and/or formulating medicines.

The different senses humans are endowed with serve different purposes. Properties of the senses of taste, smell, trigeminality, and touch (mouthfeel) are described as well as the integration of these into flavor perception. It is discussed how memories carried by these senses, which are important for food choice behavior, are distinguished from memory in a "higher" sense such as vision.

Orosensory perception is closely connected to different satiety mechanisms and reward in connection with foods and some aspects of these problems are described. Preference development and acceptance are particularly important in food perception and the mechanisms of these are explained. The same mechanisms are responsible for generation of aversions. Great care should be exercised to avoid these aversions in connection with administration of drugs.
\end{abstract}

\subsection{Introduction}

Humans are endowed with many senses which make it possible to obtain information about "what" is "where" in the environment. The senses can be divided into so-called far and near senses [1]. The far senses, vision and audition, are referred to as such because information from objects and events from far away can reach the

\footnotetext{
P. Møller ( $\bowtie)$

Faculty of Science, Department of Food Science, University of Copenhagen,

Copenhagen, Denmark

e-mail: pem@life.ku.dk 
animal via electromagnetic light waves, as is the case for vision, or via pressure waves, as is the case for audition. All other senses, the near senses, require direct physical contact with the materials these senses provide information about. The far senses are also sometimes referred to as the "higher" senses and the near senses as the "lower" senses. Memory, planning, thinking, imagery, and other cognitive processes are intertwined with the senses. The higher senses, vision and audition, lead to more elaborate cognitive processes than the lower senses. Visual and auditory imagery is a case in point, which all people are capable of. Very few people, on the other hand, claim to have olfactory imagery. Some cognitive functions, memory being the best example, pervade all senses, and olfactory, taste, and flavor memory are of crucial importance for food choice behavior [2, 3]. Besides perceptual and cognitive processes, all senses also are capable of generating emotional states and representations. Fear, anger, happiness, etc. are basic emotions, but in the context of foods the most important emotional states relate to evaluation of how well a given stimulus is liked or how disgusting it is. These evaluations, which don't need to be influenced by cognitive processes, are the main drivers for the actions which follow emotional evaluation; whether a stimulus (a food) is accepted or rejected.

Thus, all senses support perceptual, cognitive and emotional processes, but to different degrees. Some senses lend themselves more easily to cognitive processes than other senses do and some more easily represent emotional states $[4,5]$. Perceptually, the senses are, of course, phenomenologically different, i.e. smelling a substance is a purely olfactory feat and perceiving a color is brought about by activity in the visual system only. But besides these phenomenological perceptual properties of the senses, certain types of information about the environment can be extracted by more than one sense. Movement of a visible, sound emitting object can be determined by both the visual and the auditory system. The final representation in the brain of the movement of the object is a result of visuo-auditory integration processes. Even if there are only few examples of the same type of information being extracted by different senses, in the far majority of cases, objects and events present themselves to more than one sense. A certain food, e.g., has a smell, texture, and visual appearance. These different types of information all aid in the detection and identification of the food and the perceptual system integrates the different types of information. Besides providing us with a phenomenologically more interesting environment, the many senses also help us obtain more reliable and robust information about objects and events. If, for some reason, the information provided to one sense is too noisy to extract a reliable representation of the environment, information extracted by another sense will help us to reach a reliable representation. Thus, from the perspective of understanding how the sensory systems works, we do not only need to understand each individual system (the olfactory, the gustatory, the visual, etc.), but it is also crucial to understand how the senses act in concert. This is particularly the case for problems regarding food perception and appreciation as will be discussed later in connection with flavor perception.

A fair number of the senses, or sensory modalities, are of crucial importance for perception and affective evaluation of foods and for choice behavior. This has been demonstrated to rely far more on the sensory properties than upon any other parameters. 
The senses most important for food perception are, not surprisingly, those which can be activated by stimulation in the mouth: the so-called orosensory senses [6, 7]. Even though stimulation of the orosensory senses determines the sensory experience of a food, visual and auditory information can also play an important role. Vision can raise expectations of the taste and enjoyment that is about to follow, as well as prepares the body for digestion by way of the so-called cephalic reflexes.

Most of what we know about the workings of the senses in humans comes from psychophysical work $[6,7]$. Psychophysics is the science which connects the external physical world to our internal psychological states. Psychophysics studies function and functional mechanisms. It describes what the organism is capable of and which (functional) properties it has. For example, that humans can distinguish five basic tastes was originally derived from psychophysical studies. That holds for all other functional properties of the sensory systems. One might say that psychophysics maps out the functional properties of the perceptual (and cognitive and emotional) systems. Only when it has been described what the organism is capable of it is meaningful to search for neural implementations of the functions. Until recently, quite a bit of information about the localization in the brain of the different systems has come from neuropsychological patients, i.e. from patients with various brain lesions. More recently, with the advent of modern neuroimaging methods such as functional magnetic resonance imaging (fMRI), positron emission tomography (PET), electroencephalography (EEG) and magnetoencephalography (MEG), information about localization and timing of processes in the brain can be obtained from normal, healthy people. The different techniques have different virtues and drawbacks. EEG does not have the same exquisite spatial resolution as fMRI has, but it has a temporal resolution which is only limited by the speed of the electronic equipment used in an investigation. In practice, this means that EEG data has a better temporal resolution than $1 \mathrm{~ms}$. Experiments on animals, which for ethical reasons cannot be performed on humans, also contributed to describe some of the underlying neural mechanisms. But, one must always exercise care in using animal data to interpret psychophysical effects as measured in humans.

In the rest of this chapter I will discuss the orosensory senses which are most important for food perception and acceptance: taste, smell, trigeminality, and touch. I will explain what is meant by flavor and discuss some of the problems and effects of flavor. The orosensory senses are closely connected to homeostasis and reward and are crucial in determining acceptance and how (food) preferences are formed. These problems will be discussed at the end of the chapter.

\subsection{Taste}

The "taste" of a food is an important property, not the least because it accounts for most of the food choices people make. The phrase "taste" is a bit unfortunate because it gives the impression that the sense of taste completely determines the "taste" of a food. As will be made clear in this chapter, this is by no means the case. 
Limiting ourselves in this paragraph to the sense of taste (gustation), it is customary to talk of five basic tastes: sweet, salty, sour, bitter, and umami [6, 7].

The last one, umami, is less well known than the other four. It is often described as the taste of bouillon or glutamate. Umami was discovered by Japanese scientists and the name is meant to indicate "pleasurable taste," and is associated with seaweed, fermented soy, and fish products. The concept of basic tastes comprises the idea that sweet, salty, sour, bitter, and umami tastes are basic, in the sense that none of them can be obtained by any combination of the other four, and that any possible taste sensation can be created by an appropriate mixture of the five basic tastes.

The basic tastes are usually defined in terms of sucrose (sweet), quinine (bitter), sodium chloride (salty), citric acid (sour), and monosodium glutamate (umami). Different types of criticisms have been raised against the concept of basic tastes [8]. But, since the five basic tastes are used worldwide in common language and because the scientific literature on taste also uses this categorization, it will take a paradigmatic change to reorganize our knowledge about the sense of taste and at present the concept of basic tastes is very useful.

Specialized taste receptors on the tongue, palate, soft palate, and areas in the upper throat (pharynx and laryngopharynx) are activated when they come in contact with typical tastants such as alkaloids (bitter), many ionic compounds (salty), most acids (sour), sugars (sweet), and amino acids and nucleotides (umami).

Humans have about 7,500 taste buds in the mouth, each of which contains 40-60 taste cells. About two thirds of the taste buds are localized in different kinds of papillae. The rest of the taste cells are distributed in the mouth outside papillae. Taste cells interact with tastants dissolved in liquids in the mouth (water, alcohol, fats) via different receptors. Over the last 10-15 years receptor proteins for bitter, sweet, and umami have been identified. All these receptors are a subclass of the super family of G-protein-coupled receptors (GPCRs) and have been classified as T1R1, T1R2, T1R3, and T2Rs [9, 10].

Receptors for sour and salty tastes are essentially ionic channels, but the identity of the salty receptor is still speculative and controversial. Human taste cells have a lifetime of 5-30 days and are regenerated all through life.

Once a tastant has activated a taste cell, signals are transmitted to the brain for further processing. The first relay is in the nucleus of the solitary tract (NTS) in the medulla. In primates, NTS neurons transmit information to an area in the thalamus, which further connects to areas in the frontal operculum and insula. These two areas are usually referred to as the primary taste cortex [11]. These areas are connected to limbic parts of the brain and to frontal areas, notably the orbitofrontal cortex which also receives input from smell, touch, and visual areas, besides being connected to hypothalamic nuclei which hold information about hunger and satiety.

Despite the fact that we only rarely doubt whether we have added too much salt to a dish or too much tonic in our gin and tonic, or if our coffee is too bitter, the underlying neurophysiological processes that allow us to immediately evaluate these questions are very complicated. We can experience the character of a taste (sweet, salty, sour, etc.), but we can also distinguish different intensities of tastes. Since a taste cell contains receptors for the different basic tastes it can respond to 
more than a single basic taste. There is therefore not enough information in the signal from a single taste cell to determine the stimulus. The cell could relay the same signal to the brain when stimulated with a weak sweet solution as when stimulated with a strong bitter solution. Another taste cell with another distribution of receptors will in general respond differently to the weak sweet and strong bitter solutions. There will, on the other hand, be other concentrations of basic tastes which this cell will respond to in the same way. If, however, we consider the distribution of activities in a sufficient number of taste cells, it is possible to determine the character and strength of the taste stimulus. The character and intensity of tastes are thought to be coded by activities in many taste cells. This is referred to as distributed coding.

Outside the laboratory the human gustatory system rarely encounters single basic tastes. Most food stimuli are complex mixtures of chemicals which activate a number of basic tastes simultaneously and there are important interactions between the different tastes in a mixture of tastes [12]. Tastes can suppress or enhance each other. Masking, the nonlinear process in which the addition of compound $\mathrm{A}$ decreases the intensity of compound $\mathrm{B}$ in a manner that goes beyond linear reductions in intensity, is of great interest to mask bad tastes of pharmacological compounds. This problem is dealt with in Chap. 9 by Charles R. Frey. Mixture interactions at low concentration which generate suppressions in some cases and enhancement in other can be used to modulate the flavor of a food. The effect of salt in breads or in French pancakes is a well-known example. A well-tasting bread or pancake does not taste salty, but following the same recipes for these foods without the salt leads to breads and pancakes which are unpalatable to most people.

The five basic tastes are not sufficient to create all of the thousands of different "tastes" available to us from different foods. Smelling a food gives an impression of its so-called aroma. The sense of smell therefore seems to be important for the perception of foods. Think about how food tastes when you have a common cold, or how wine tastes if you block your nose while drinking it. If smell was not important for the "taste" of food it should be possible to "create" the "taste" of, e.g., an orange, by a particular mixture of the basic tastes - and this is not possible. The sensation of smell produces an almost infinite number of possible "tastes" when combined with the other senses which contribute to the perception of foods. The dimension of the space describing the different possible "tastes" increases enormously from the five dimensions that the sense of taste provides on its own.

\subsection{Sense of Smell}

Stevenson [13] has identified three major classes of function of human olfaction: in ingestive behaviors (e.g., food detection and evaluation, appetite regulation, breast finding), in avoiding environmental hazards (e.g., fear related, disgust related), and in social communication (e.g., reproductive behaviors and emotional contagion). He also stresses the importance of learning in human olfaction and he points out that 
learning and memory in olfaction are distinguished from learning in other sensory modalities by its speed, its resistance to extinction and its often implicit nature. Besides the three classes of function of human olfaction listed by Stevenson, human odor memory supposedly also plays a large role in generating feelings of being at home and of well-being. Furthermore, "Proustian effects," i.e. the ability of odors and odor memory to open up rich recollections and feelings of times and events gone by are important roles of odor memory.

\subsection{Structure of the Olfactory System}

The receptors for the sense of smell are located in the nose and occupy $4-5 \mathrm{~cm}^{2}$ in each nostril. It is estimated that humans have about 350 different types of receptor cells. It is noteworthy that this number of different cells is dramatically different from the number of different receptor cells we have in the eye (three types (cones) for day vision and one type (rod) for night vision/vision under low ambient luminance conditions). This difference strongly suggests that coding and processing of visual and olfactory information use different strategies. Most odorants activate many of these 350 different cells, but different smells activate different subsets to different degrees. From the receptor cells in the nose signals are transmitted to neurons in the olfactory bulbs, bulb-formed neural structures located behind the eyes. After signal transformations in the bulbs, olfactory information is relayed via the olfactory tract to the piriform cortex, the so-called primary olfactory cortex, and to amygdaloid areas. The amygdalae (one in each side of the brain) are part of the limbic system, which is strongly implicated in emotional processing. This means that olfactory information reaches emotional brain areas after only two relays. Signals in other senses need to pass more relay stations before reaching the amygdalae and other emotional brain areas. This strongly suggests that the sense of smell has particular salience as a conveyer of emotions. This suggestion has been supported by a number of investigations $[14,15]$. From the piriform cortex and amygdaloid areas, signals are relayed to other areas in the brain, both in the midbrain and in the frontal areas of the brain. Various frontal areas have been implicated in olfactory processing, most notably the orbitofrontal cortex. The olfactory brain is ipsilaterally organized, as opposed to the other senses, which are contralaterally organized. Ipsilateral means that signals from the right nostril are transmitted to the same (ipsi) side, i.e. the right side; and signals from the left nostril are transmitted to the left side of the olfactory brain. In a contralateral sense (contra meaning opposite) such as vision, signals from the left visual field are transmitted to the right part of the visual brain and vice versa for signals from the right visual field. Olfaction is also distinguished from the other senses in that olfactory signals do not have to pass through a midbrain relay in the thalamus, as signals in the other senses do, before they are transmitted to limbic areas and to areas in the orbitofrontal cortex. From an anatomical point of view olfaction is wired very differently than the other senses. This, together with the dramatic differences in number of different types of receptor 
Table 8.1 Comparison of important properties of the senses of vision, audition, olfaction, gustation, touch, and pain

\begin{tabular}{lllllll}
\hline & Vision & Audition & Olfaction & Gustation & Touch & Pain \\
\hline Characteristic involved & & & & & & \\
Strict intersubjectivity & Yes & Yes & No & No & Yes? & No \\
Inborn properties & Yes & Yes & No & (Yes) & Yes & Yes \\
Directional perception & Yes & Yes & No & No & Yes? & Yes temporal \\
Relative perception & Yes & Yes & No & No & Yes? & Yes \\
Intensity discrimination & High & High & Poor & Poor & High & High/low \\
Quality discrimination & High & Very high & Very high & Moderate & High & High \\
Absolute sensitivity & High & High & High & Moderate & High & High? \\
Adaptation & Partial & Low & Complete & Partial & Complete & Sensitization \\
In focus of attention & Mostly & Often & Seldom & Seldom & Sometimes & Mostly \\
\hline
\end{tabular}

Note how the "higher senses" vision and audition are distinguished from olfaction and gustation. The lower senses olfaction and gustation are seldom in the focus of attention and has very little intersubjectivity, but are more prone to adaptation than vision and audition. Courtesy of E.P. Köster

cells (in, e.g., vision and olfaction), suggests that olfaction has very different properties than, e.g. vision. This anatomically based suggestion is supported by functional comparisons as illustrated in Table 8.1.

\subsection{Foods and the Sense of Smell}

The sense of smell is crucial for the "taste" of foods. Think about how food "tastes" when your nose is blocked. All foods with other "tastes" than pure sweetness, sourness, saltiness, umami, or bitterness contain aroma substances which are released when the food is chewed and brought to smell receptors in the nose via the nasopharynx which connects the mouth and the nose. Without odor perception our experience of foods would be very limited. People who have lost the sense of smell describe how foods have become boring and how their enjoyment of meals has disappeared. Hedonic value, i.e. whether an odor smells good or bad is by far the most important property of an odor. This holds true both for its role in ingestive behaviors and when it helps us to avoid environmental hazards.

Smell serves us in two ways when we deal with foods. Before we ingest a food we often smell it by sniffing at it. This sniffing behavior allows us to judge if the food is safe to ingest, and we form anticipations of the quality and taste of it. When a smell is estimated or appreciated by sniffing, odorants enter the nose through the nostrils. This type of olfactory perception, called orthonasal perception, also helps to prepare the body for ingestion by means of the so-called cephalic reflexes, such as increasing the flow of saliva in the mouth, increasing the release of insulin in the pancreas and increasing the release of acid in the stomach. The very sight of the food to be eaten also triggers cephalic reflexes [16, 17].

Once we have decided that the food we estimate by smelling is safe and (probably) well-tasting, we ingest it, chew it, and swallow it. During chewing and swallowing, 
aroma molecules are released from the food and these reach the olfactory epithelium via the nasopharynx. Olfactory perception via this route is called retronasal olfactory perception. These different functions of olfactory perception seem to be reflected in the neural underpinnings of anticipatory and consummatory food chemosensation. Small and colleagues have found separable and overlapping representations of anticipatory and consummatory chemosensation $[18,19]$.

There is no strict intersubjectivity in olfaction, see Table 8.1. Olfactory experiments often require at least 20 subjects, whereas $3-5$ subjects are usually enough to obtain sufficient statistical power in vision experiments. Olfactory judgment of pleasantness of odors is learned behavior. This is particularly important for the perception and evaluation of foods and explains why very different culinary traditions have developed in different parts of the world based upon what nature has to offer at particular places. We come to like what we have access to.

Olfactory adaptation, i.e. that sensitivity to a stimulus is reduced when we are exposed to it, is very strong and sometimes complete. These effects can be quite quick, rendering an odorant unnoticeable within a few seconds. There are both peripheral (in the nose) adaptation mechanisms and central (in the brain) mechanisms. Furthermore, an odorant does not only adapt the olfactory system's ability to detect the presence of itself (auto-adaptation) but may also affect the system's perception of other odorants (cross-adaptation). These effects strongly affect what we perceive when we engage in continual eating or drinking. If a substance with an unwanted flavor is present in a drink, for example, but is not perceived because it is masked by another flavor component, adaptation to the masker could lead to perception of the unwanted flavor. This might be why the second beer often tastes different from the first you drink.

Smells are seldom in our focus of attention, but this does not mean they do not serve to guide behavior. Recent work has revealed that odors and flavors that are not attended to at all are nevertheless encoded and remembered [20-23]. This type of "incidental" learning builds memories which are less explicit and declarative than memories of a more semantic nature. In our daily dealings with foods we seldom have any intention of encoding what we encounter. The memories we form of such events are of a much more implicit nature and learning is incidental. We nevertheless do remember events and objects even without any intention to do so.

Incidentally learned information about odors and flavors is not based on actual recognition of a certain target, but rather on detection of novelty [24]. That is, when prompted, we detect that a certain stimulus is not identical to the one we encountered previously. When presented with the same stimulus as previously (the target) we are often at chance level at detecting that it is the same stimulus. These effects have also been found in other lower senses than smell [21, 25-27]. Furthermore, in contrast to what is usually found in memory experiments, in the incidental learning experiments on the lower senses referred to above, elderly people remember stimuli as well as young people. These results are relevant to the understanding of eating behavior and choice of foods. They might also help to explain the relative constancy of food preferences over a lifetime despite the rather dramatic changes of the sensory systems with age. 
Relying on memory systems with these properties in conjunction with senses which are sensitive to expectation and anticipation effects, that is, senses which will incorporate top-down information into the formation of a percept, might be sufficient to explain the relative constancy of food preferences over life.

Since olfactory stimuli are rather poor in information content compared to, e.g., visual stimuli one might expect that olfaction would be more prone to influences from top-down effects. Such effects have indeed been found in olfaction. Different expectations to an odor dramatically change the activities in the olfactory system [28-30].

\subsection{Sense of Trigeminality}

Trigeminal stimuli are occasionally referred to as "irritants," since the sensations they give rise to can be unpleasant or even painful. Besides allowing us to perceive hot spices (chili, garlic, mustard, horseradish, ginger, etc.) and $\mathrm{CO}_{2}$ in fizzy drinks, most chemical substances will also activate the trigeminal system at sufficiently high concentration. This sense is also known as chemesthesis and stimuli which activate the trigeminal system in the mouth are often said to have "oral pungency" [31]. The receptors of the trigeminal system consist of the so-called free nerve endings. These receptors are found in the mouth, the nose, the throat, and around the eyes. When a (food or other) substance scratches in the nose or mouth we experience a trigeminal sensation. It is important to note that trigeminal sensation is not part of the olfactory or gustatory system, but constitutes a separate sense. Trigeminal signals are relayed from the sensory periphery to the brain by the 5 th cranial nerve, the trigeminal nerve. Other painful sensations such as cold and heat are also sensed by the trigeminal system as is the physical temperature in the mouth and the cooling effects of menthol and other substances. Many of the functional properties of this system are very different from those of olfaction (smell) and gustation (taste). For example, oral pungency typically has a slow onset but can persist for prolonged periods, minutes to tens of minutes.

This is contrary to the sense of taste, which is most intense for the few seconds the food is in the mouth. Also, trigeminal stimuli might not only adapt (de-sensitize) the system but also induce higher sensitivity to stimuli [32]. These differences in the temporal nature of pungency and taste is of great interest when considering the palatability of foods and the overall satiety they provide. In many cases, the longterm effects of pungency will make foods both more palatable and more satiating.

Without pungency many foods would be bland; imagine horseradish without the heat or garlic with no bite. Clearly, trigeminal sensation plays a crucial role in our evaluation of the palatability of foods.

Trigeminal stimuli also seem to have very interesting effects on metabolism and satiety. It has been reported that trigeminal stimuli can increase fat metabolism [33, $34]$ and increase satiety $[35,36]$. Thereby, by both mechanisms, they potentially provide a contribution to curb the accelerating obesity epidemic. 


\subsection{Mouthfeel, Sense of Touch}

Olfactory, gustatory, and trigeminal sensations are determined by the submicroscopic (molecular) properties of the stimulants. Mouthfeel, on the other hand, is what a food feels like in the mouth. It depends on the macroscopic (and mesoscopic) properties of the food. Properties such as thickness, viscosity, hardness, elasticity, and brittleness are judged by how they feel in the mouth, not by whether they might also have a taste or a smell. These sensations are mostly conveyed by a tactile or touch sensation. Humans have a number of receptors for tactile stimulation with different spatial and temporal sensitivities which relay signals to the somatosensory cortex for further processing of tactile information. In connection with foods it is customary to talk of "texture perception." Not all types of texture perception are, however, only determined by the sense of touch. Therefore, Szczesniak [37] has defined texture as "...the sensory and functional manifestation of the structural, mechanical and surface properties of foods detected through the senses of vision, hearing, touch and kinesthetics."

Thus, it is not only touch which provides the sensation of the texture of a food. Audition and kinesthesis are also contributing to the perception of the texture of a food, as exemplified by the crunchy sounds produced when chewing (fresh) corn flakes.

Human sensitivity to texture under laboratory conditions is very high. Perception of solid particles in a solution is so sensitive that they do not go unnoticed before they are smaller than $3 \mu \mathrm{m}$ in diameter. This has been exploited commercially in a number of fat replacers and mimetics [38] where spherical microparticulates in the range of $0.1-3 \mu \mathrm{m}$ are the main functional ingredient. Particles this small are perceived as smooth and may contribute to creaminess.

There is a marked difference between the food that enters the mouth and the wetted bolus which is swallowed later. Different foods follow different pathways during oral handling with respect to degree of structure, degree of lubrication over time, or number of chews.

The texture of a food changes during consumption. The saliva lubricates the food, and enzymes in the saliva affect the viscosity of semisolids and liquids. Problems with saliva production are not uncommon in elderly people and in some neurological diseases. These lubrication problems can cause severe problems with food bolus formation and swallowability of foods.

\subsection{Flavor: Integration of Sensory Information}

As explained above, many senses contribute to the taste of foods. Even though a number of separate senses contribute to each food sensation, we do not perceive foods as a number of individual sensations, but rather as a coherent (integrated) 
whole. Integration of different sensory modalities is commonplace in the sensory system. When you watch a film in a movie theater you perceive the sounds/speech as coming from the actors' mouths, even though the sound is produced by loudspeakers located elsewhere in the movie theater. Information from your visual and auditory systems is integrated centrally in the brain to integrate the different types of sensory information into coherent meaningful wholes.

Much the same happens when we perceive any of the many tastes (flavors) available to us. Flavor is normally defined to be the perception that results from taste, smell (retronasal), trigeminality, and touch (mouthfeel) when a food is eaten [39] and is perhaps the most multimodal of all of our sensory experiences. Visual and auditory information can influence the flavor perceived when eating a food, but these influences are mostly exerted by creating expectations based on prior associations and these types of information are therefore normally not included in the multimodal flavor concept.

The binding of different sensations into coherent wholes takes place within individual senses as well as between different senses. Most food aromas (smells) are mixtures of hundreds of different types of molecules with their own smells. These individual smells are in general completely disguised by the integration into an overall smell of the food. It has been demonstrated that humans are not capable of identifying more than two to three components correctly in mixtures of smells [40]. That is, if a mixture consists of more than three components, we cannot reliably report what these components are. The mixture, of course, still has a characteristic smell. But it is a synthetic perception, which is the result of olfactory integration processes. They abolish perception of the smell of the individual components. These integration processes are of crucial importance for the perception of foods. Nevertheless, a smell which is unpleasant on its own can, when added to a mixture of other odorants, turn this into a more pleasantly smelling mixture. An odorant present in a mixture in such a small concentration that it cannot be detected if it was presented alone can change and improve the overall pleasantness of the mixture. These effects are well known and used in the world of fragrances.

Integration of the different senses into an overall flavor percept has been studied both by psychophysical and by neuroimaging means. Verhagen and Engelen [41] have collected and described many important effects in multimodal food perception. Neuroimaging studies conducted on flavor perception have revealed that a number of brain areas are involved in the integration of signals from the different food-related senses. These include anterior ventral insula, anterior cingulate cortex, amygdala and most notably the orbito-frontal cortex, located in the front of the brain over the orbits of the eyes [39, 42]. This area receives input from smell, taste, touch and vision, and besides being part of a putative flavor circuit in the human brain, the orbito-frontal cortex has also been implicated as the area that computes and represents sensory-specific satiety [43], the well-known phenomenon that liking of a particular food decreases with the amount eaten, without affecting appreciably the liking of other foods. 


\subsection{Reward and Homeostasis}

When we eat and drink we become satiated, and intake normally ends. Much of satiety is controlled by a set of the so-called homeostatic processes, which are negative feedback loop processes that help keep appropriate balances of nutrients in the body.

Hunger is signaled by a number of hormonal substances such as ghrelin in the stomach and NPY, orexin, and AgRP in the hypothalamus. Different nuclei in the hypothalamus are thought to control hunger and satiety and the associated relevant behaviors. Intake of food depresses the hunger signals and leads to an increase in satiety signals such as cholecystokinin (CCK), glucagon-like peptide-1 (GLP-1), peptide YY (PYY), insulin, and leptin. Besides the homeostatic satiety processes, humans also possess the so-called sensory-specific satiety mechanisms as mentioned above. An animal endowed with such mechanisms will tend to eat a varied diet, which in turn will counteract the risk of malnutrition. These mechanisms obviously have much influence in guiding food choices during a meal. These mechanisms are not innate, as demonstrated recently [44]. It was found that sensory-specific satiety in children is coupled strongly to the eaten product, whereas clear transfer effects were found in adults. That is, for adults, eating a food with a certain sensory profile will lead to some transfer of decline in liking to other foods that share one or more characteristic sensory attribute with the food eaten to satiation.

These mechanisms also highlight the importance of reward for food intake. Reward mechanisms are emotional in nature, and these mechanisms might have evolved to guarantee engagement in behaviors important for survival. A varied energy supply is necessary for survival, and eating food in most cases leads to rewarding feelings and pleasure $[45,46]$. Dopaminergic pathways in the brain, i.e., neural structures depending on dopamine as neurotransmitter, have long been known to be crucial for reward mechanisms [47]. Recently, however, a new neurology of reward has emerged in which reward is suggested to consist of distinguishable processes in separable neural substrates. In this account liking (emotion or affect) is separated from wanting (or motivation), each having explicit as well as implicit components. Explicit processes can come to awareness, whereas implicit processes exert their influence without being conscious to us [48, 49]. Contrary to previous belief, the pleasure of eating palatable food is not mediated by dopamine but rather by opioid transmission in a neural network including the nucleus accumbens, ventral pallidum, parabrachial nucleus, and nucleus of the solitary tract. Wanting (appetite, incentive motivation), on the other hand, is suggested to rely on a dopaminergic system with projections from the ventral tegmental area to the nucleus accumbens and circuits involving areas in the amygdala and prefrontal cortex [48]. The distinction between liking and wanting was originally based on work on rodents [48], but psychophysical and neuroimaging studies on humans support the distinction $[49,50]$.

It is reasonable to include learning processes in the set of processes that we need to understand reward. This is particularly important with respect to foods where 
almost all liking and wanting are results of learning processes. Since eating is crucial to survival the motivational mechanisms and rewards related to feeding are strong. It might therefore not be very surprising that the biological mechanisms of feeding and addiction overlap throughout evolutionary history. Work in rodents has demonstrated increases in dopamine in the nucleus accumbens induced by food and by amphetamine. The dopamine response to the two types of stimulation is qualitatively identical, although the size of the response is an order of magnitude larger for amphetamine [51]. Similar results have been obtained from neuroimaging studies on humans $[52,53]$. Besides dopaminergic systems, several cholinergic systems in the brain have been implicated in both food and drug intake [54]. Berthoud [46] has argued that human food intake control, in the obesogenic environment of affluent societies, is guided by cognitive and emotional processes rather than by homeostatic processes. The hypothalamic system, classically believed to control food intake, has an abundance of connections to other parts of the brain involved in sensory and reward processing, and evidence suggests that these cortico-limbic processes can dominate the homeostatic regulatory circuits in the hypothalamus.

\subsection{Preferences and Acceptance}

The foods we eat are to a large extent determined by our preferences. Other factors such as price and social context are also important, but within the constraints set by these factors, we eat what we prefer or like.

Research has demonstrated that we are born with very few specific preferences [55]. Newborn babies have a strong preference for sweet and fatty taste and a dislike for bitter taste. From a developmental point of view the preference for sweetness and fat facilitate breastfeeding. The dislike for bitter has been interpreted as an inborn defense against bitter-tasting toxic alkaloids in nature. Most people have to reach adulthood before they have learned to appreciate the bitter taste of beer, coffee, and many vegetables. Besides these few examples, all other preferences are incidentally learned by exposure to the foods of one's culture. This allows man to be omnivorous and able to adapt to whatever eatable materials are found in the environment. There are no fundamental differences between the nervous systems of different human races, but different cultures have nevertheless developed radically different cuisines or food cultures based on what nature provides. This demonstrates very clearly that food preferences are learned and not genetically inherited.

Learning starts in the fetal state [56] and during breastfeeding [57] and continues through childhood and later life. Flavor learning in the fetal state is believed to take place via transfer of flavor substances eaten by the mother to the amniotic fluid and from there to the developing sense organs of the fetus. After birth, and probably all through life, a number of the so-called conditional learning mechanisms act to change food preferences. Conditional learning means that we learn, or change, on the condition that another unconditional stimulus is present together with the stimulus we learn about, the conditional stimulus. In the case of preference learning an 
already well-liked stimulus plays the role of the unconditional stimulus, which is a stimulus to which we have an unconditional positive response. The response to the conditional stimulus presented alone is called the conditional response. For a new food, or other substance not resembling anything ever encountered before, the conditional response will most often be a rejection. If, however, the new food is presented together with a stimulus with an already liked taste or positive bodily effect, it turns out that after a few presentations of the conditional and unconditional stimulus together, people will respond differently to the conditional stimulus when it is presented alone. What initially was rejected and not liked will now be accepted and liked. It is as if the properties of the unconditional stimulus have been taken over by the conditional stimulus. If a known liked flavor is paired with a novel flavor, the conditional learning is called flavor-flavor learning. Another type of conditional learning important for changes of preferences for foods is the so-called flavor-nutrient learning. In this type of learning it is the nutrient value/energy in an unconditional stimulus which, when paired with the novel flavor of a conditional stimulus, will increase the liking of the novel flavor.

The so-called mere exposure effect, where a number of exposures to a new flavor changes appreciation of it, is also an important mechanism for preference development $[58,59]$. Mere exposure might be a result of conditional learning. In this interpretation, it is the absence of adverse effects, after having been exposed to a novel flavor, which plays the role of the unconditional stimulus [60].

These forms of preference formation mechanisms are believed to be important in forming children's food preferences. They play undoubtedly also a role in the changes of preferences experienced by adults, but for this population it is not as well defined to talk of novel flavors, since a novel flavor might have certain resemblances to other flavors that have been perceived previously. Even though food preferences do change, it is interesting that preferences developed in childhood seem to be quite long-lasting [61]. Whether this is also the case for food or taste aversions is an important question. A food aversion is a strong dislike of a certain taste or flavor. It comes about as a result of conditional learning. If a taste has been experienced in temporal proximity to an adverse effect or illness, an aversion [58] might develop to the taste even though the adverse effect is not causally related to the flavor. Being infected with a stomach infection that eventually causes pain and vomiting will often lead to an aversion of the flavor of the food one consumed while still feeling well, even though the illness is not related to consumption of the food. This type of aversion effect should be considered when administering drugs with known adverse effects. There is a serious risk that the patient will develop aversions to foods eaten in close proximity to the adverse effects. Using flavors to mask "bad taste" of a drug should avoid flavors which are common and important in the patient's food culture. There is a strong need for research into development of food aversions in children who are treated with medicines. Do some flavors in combination with some medicines present less risk of generating aversion than they do with other medicines? It might also be that some food flavors are less amenable to aversion effects than others. Such flavors should be used wherever they are appropriate. If it is not 
possible to completely avoid aversion effects, it is important to learn which flavor aversions will have the shortest lifetime and to devise methods to extinguish flavor aversions.

\section{References}

1. Köster EP (2002) The specific characteristics of the sense of smell. In: Rouby C, Schaal B, Dubois D, Gervais R, Holley A (eds) Olfaction, taste, and cognition. Cambridge University Press, Cambridge

2. Morin-Audebrand L, Mojet J, Chabanet C, Issanchou S, Møller P, Köster EP, Sulmont-Rossé C (2012) The role of novelty detection in food memory. Acta Psychol 139:233-238

3. Köster EP, Mojet J (2007) Theories of food choice development. In: Frewer L, Van Trijp HCM (eds) Understanding consumers of food products. Woodhead, Cambridge

4. Herz RS (2002) Influences of odors and mood on affective cognition. In: Rouby C, Schaal B, Dubois D, Gervais R, Holley A (eds) Olfaction, taste, and cognition. Cambridge University Press, Cambridge

5. Herz RS (2002) An examination of objective and subjective measures associated to odors, music and paintings. Empirical Stud Arts 16:137-152

6. Doty RL (ed) (2003) Handbook of olfaction and gustation, 2nd edn. Marcel Dekker, New York

7. Firestein S, Beauchamp GK (eds) (2008) The senses: a comprehensive reference, vol 4, Olfaction and taste. Academic/Elsevier, Oxford

8. Delwiche J (1996) Are there 'basic' tastes? Trends Food Sci Technol 7:411-415

9. Chaudhari N, Roper SD (2010) The cell biology of taste. J Cell Biol 190:285-296

10. Dubois GE, Desimone J, Lyall V (2008) Chemistry of gustatory stimuli. In: Firestein S, Beauchamp GK (eds) The senses: a comprehensive reference, vol 4, Olfaction and taste. Academic/Elsevier, Oxford

11. Rolls ET, Scott TR (2003) Central taste anatomy and neurophysiology. In: Doty RL (ed) Handbook of olfaction and gustation, 2nd edn. Marcel Dekker, New York

12. Breslin PAS (1996) Interactions among salty, sour and bitter compounds. Trends Food Sci Technol 7:390-399

13. Stevenson RJ (2010) An initial evaluation of the functions of human olfaction. Chem Senses 35:3-20

14. Herz RC, Cupchik GC (1995) The emotional distinctiveness of odor-evoked memories. Chem Senses 20:517-528

15. Herz RC, Eliassen J, Beland S, Souza T (2004) Neuroimaging evidence for the emotional potency of odor-evoked memory. Neuropsychologia 42:371-378

16. Giduck SA, Threatte K (1987) Cephalic reflexes: their role in digestion and possible roles in absorption and metabolism. J Nutr 117:1191-1196

17. Mattes RD (1997) Physiologic responses to sensory stimulation by food: nutritional implications. J Am Diet Assoc 97:406-413

18. Small DM, Gerber JC, Mak YE, Hummel T (2005) Differential neural responses evoked by orthonasal versus retronasal odorant perception in humans. Neuron 47(4):593-605

19. Small DM, Veldhuizen MG, Felsted J, Mak YE, Mcglone FC (2008) Separable substrates for anticipatory and consummatory food chemosensation. Neuron 57:786-797

20. Møller P, Wulff C, Köster EP (2004) Do age differences in odour memory depend on differences in verbal memory? Neuroreport 15(5):915-917

21. Møller P, Mojet J, Köster EP (2007) Incidental and intentional flavour memory in young and older subjects. Chem Senses 32(6):557-567

22. Sulmont-Rossé C, Møller P, Issanchou S, Köster EP (2008) Effect of age and food novelty on food memory. Chem Percept 1:199-209 
23. Köster EP (2005) Does odour memory depend on remembering odours? Chem Senses 30(Suppl 1):i236-i237

24. Møller P, Köster EP, Dijkman N, de Wijk R, Mojet J (2012) Same-different reaction times to odors: some unexpected findings. Chem Percept 5:158-171

25. Mojet J, Köster EP (2005) Sensory memory and food texture. Food Qual Preference 16:251-266

26. Köster MA, Prescott J, Köster EP (2004) Incidental learning and memory for three basic tastes in food. Chem Senses 29:441-453

27. Laureati M, Morin-Audebrand L, Pagliarini E, Sulmont-Rossé C, Köster EP, Mojet J (2008) Food memory and its relation with age and liking: an incidental learning experiment with children, young and elderly people. Appetite 5:273-282

28. de Araujo IE, Rolls ET, Velazco MI, Margot C, Cayeux I (2005) Cognitive modulation of olfactory processing. Neuron 46:671-679

29. Grabenhorst G, Rolls ET, Bilderbeck A (2008) How cognition modulates affective responses to taste and flavour: top-down influences on the orbitofrontal and pregenual cingulated cortices. Cereb Cortex 18:1549-1559

30. Grabenhorst G, Rolls ET (2008) Selective attention to affective value alters how the brain processes taste stimuli. Eur J Neurosci 27:723-729

31. Doty RL, Cometto-Muniz JE (2003) Trigeminal chemosensation. In: Doty RL (ed) Handbook of olfaction and gustation, 2nd edn. Marcel Dekker, New York

32. Green BG (1996) Chemesthesis: pungency as a component of flavor. Trends Food Sci Technol 7:415-423

33. Belza A, Frandsen E, Kondrup J (2007) Body fat loss achieved by stimulation of thermogenesis by a combination of bioactive food ingredients: a placebo controlled, double-blind 8-week intervention in obese subjects. Int J Obes 31(1):121-130

34. Westerterp-Plantenga MS, Diepvens K, Joosen AMCP, Berube-Parent S, Tremblay A (2006) Metabolic effects of spices, teas, and caffeine. Physiol Behav 89(1):85-91

35. Reinbach HC, Smeets A, Martinussen T, Møller P, Westerterp-Plantenga MS (2009) Effects of capsaicin, green tea and $\mathrm{CH}-19$ sweet pepper on appetite and energy intake in humans in negative and positive energy balance. Clin Nutr 28:260-265

36. Westerterp-Plantenga MS, Smeets A, Lejeune MPG (2005) Sensory and gastrointestinal satiety effects of capsaicin on food intake. Int J Obes (Lond) 29(6):682-688

37. Szczesniak AS (2002) Texture is a sensory property. Food Qual Preference 13:215-225

38. Kilcast D, Clegg S (2002) Sensory perception of creaminess and its relationship with food structure. Food Qual Preference 13:609-623

39. Small DM (2012) Flavor is in the brain. Physiol Behav. doi:10.1016/j.physbeh.2012.04.011

40. Jinks A, Laing DG (1999) A limit in the processing of components in odour mixtures. Perception 28:395-404

41. Verhagen JV, Engelen L (2006) The neurocognitive bases of human multimodal food perception: sensory integration. Neurosci Biobehav Rev 30:613-650

42. Rolls ET (1999) The brain and emotion. Oxford University Press, Oxford

43. Kringelbach ML, O’Doherty J, Rolls ET, Andrews C (2003) Activation of the human orbitofrontal cortex to a liquid food stimulus is correlated with its subjective pleasantness. Cereb Cortex 13:1064-1071

44. Olsen A, Ritz C, Hartvig DL, Møller P (2011) Comparison of sensory specific satiety and sensory specific desires to eat in children and adults. Appetite 57:6-13

45. Berridge KC (1996) Food reward: brain substrates of wanting and liking. Neurosci Biobehav Rev 20:1-25

46. Berthoud H-R (2004) Mind versus metabolism in the control of food intake and energy balance. Physiol Behav 81:781-793

47. Wise RA (2002) Brain reward circuitry: insights from unsensed incentives. Neuron $36: 229-240$

48. Berridge KC, Robinson TE (2003) Parsing reward. Trends Neurosci 26:507-513 
49. Finlayson G, King N, Blundell JE (2007) Liking vs. wanting food: importance for human appetite control and weight regulation. Neurosci Biobehav Rev 31:987-1002

50. Small DM, Zatorre RJ, Dagher A, Evans AC, Jones-Gotman M (2001) Changes in brain activity related to eating chocolate. Brain 124:1720-1733

51. Bassareo V, Di Chiara G (1999) Modulation of feeding-induced activation of mesolimbic dopamine transmission by appetitive stimuli and its relation to motivational state. Eur $\mathbf{J}$ Neurosci 11:4389-4397

52. Volkow ND, Wise RA (2005) How can drug addiction help us understand obesity. Nat Neurosci 8:555-561

53. Wang GJ, Vollow ND, Logan J, Pappas NR, Wong CT, Zhu W et al (2001) Brain dopamine and obesity. Lancet 357:354-357

54. Avena NM, Rada P, Hoebel BG (2008) Evidence for sugar addiction: behavioral and neurochemical effects of intermittent, excessive sugar intake. Neurosci Biobehav Rev 32:20-39

55. Birch LL (1999) Development of food preferences. Annu Rev Nutr 19:41-62

56. Schaal B, Marlier L, Soussignan R (2000) Human foetuses learn odours from their pregnant mother's diet. Chem Senses 25:729-737

57. Mennella JA, Jagnow CP, Beauchamp GK (2001) Prenatal and postnatal flavor learning by human infants. Pediatrics 107:1-6

58. Schafe GE, Bernstein IL (2001) Taste aversion learning. In: Capaldi ED (ed) Why we eat what we eat, 3rd edn. American Psychological Association, Washington

59. Hausner H, Olsen A, Møller P (2012) Mere exposure and flavour-flavour learning increase 2-3 year-old children's acceptance of a novel vegetable. Appetite 58:1152-1159

60. Zajonc RB (2001) Mere exposure: a gateway to the subliminal. Curr Dir Psychol Sci $10: 224-228$

61. Nicklaus S, Boggio V, Chabanet C, Issanchou S (2004) A prospective study of food preferences in childhood. Food Qual Preference 15:805-818 